Supporting Information for

\title{
Molecular Insight into the Binding Property and Mechanism of Sulfamethoxazole to Extracellular Protein of Anammox Sludge
}

Gui-Feng Li, ${ }^{\dagger}$ Wen-Jie Ma,${ }^{\dagger}$ Zhi-Qi Ren,${ }^{\dagger}$ Ye Wang,${ }^{\dagger}$ Jing-Peng Li,,${ }^{\dagger}$ Jia-Wen Zhao, ${ }^{\dagger}$ Shu-Ting Li, ${ }^{\dagger}$ Qi Liu,${ }^{\dagger}$ Ye-Nan Gu, ${ }^{\dagger}$ Ya-Fei Cheng, ${ }^{\ddagger}$ Bao-Cheng Huang ${ }^{\dagger *}$, Ren-Cun $\operatorname{Jin}^{\dagger *}$

Laboratory of Water Pollution Remediation, School of Life and Environmental Sciences, Hangzhou Normal University, Hangzhou 311121, China

tDepartment of Environmental Science and Engineering, Fudan University, Shanghai 200438, China

\section{*Corresponding Authors}

Bao-Cheng Huang - Laboratory of Water Pollution Remediation, School of Life and Environmental Sciences, Hangzhou Normal University, Hangzhou 311121, China; orcid.org/0000-0001-7074-4089; Email: huangbc@,hznu.edu.cn

Ren-Cun Jin - Laboratory of Water Pollution Remediation, School of Life and Environmental Sciences, Hangzhou Normal University, Hangzhou 311121, China; Email: rcjin@hznu.edu.cn 


\section{SUPPORTING METHODS}

Proteomic Analysis of EPSs. Proteins were extracted from the anammox EPSs, and the concentration was determined by the Bradford method. After the sample quality evaluation via sodium dodecyl sulfate polyacrylamide gel electrophoresis, the protein samples were processed via reductive alkylation. Afterwards, proteins were subjected to trypsin digestion, iTRAQ labeling, and vacuum-drying. The liquid chromatography coupled with tandem mass spectrometry analysis was finally performed according to the method described by Chen et al. ${ }^{1}$ All raw files were searched with Proteome Discoverer version 2.4. 


\section{Text S1. The double reciprocal formula}

$\frac{1}{A-\mathrm{A}_{0}}=\frac{1}{\mathrm{a} \cdot \mathrm{K} \cdot \mathrm{C}}+\frac{1}{\mathrm{a}}$

$A_{0}$ and $A$ represented the absorbance before and after adding SMX, $C$ was the

concentration of SMX, and $a$ was constant.

\section{Text S2. Thermodynamic formulas}

$$
\begin{aligned}
& \ln \frac{\mathrm{K}_{2}}{\mathrm{~K}_{1}}=\frac{\Delta H}{\mathrm{R}}\left(\frac{1}{\mathrm{~T}_{1}}-\frac{1}{\mathrm{~T}_{2}}\right) \\
& \Delta G=\Delta H-\mathrm{T} \Delta S=-\mathrm{RT} \ln \mathrm{K}
\end{aligned}
$$

Where, $\mathrm{T}$ was the thermodynamic temperature; $\mathrm{R}$ was 8.31 atmospheric pressure, $\mathrm{T}_{1}$ and $T_{2}$ were the thermodynamic temperatures under two states, and $K_{1}$ and $K_{2}$ were the binding constants under the corresponding two temperature conditions.

\section{Text S3. Stern-Volmer formula}

$\frac{F_{0}}{F}=1+K_{s v}\left[Q_{t}\right]=1+k_{q} \tau_{0}\left[Q_{t}\right]$

$F_{0}$ and $F$ were fluorescence intensities of each EPSs component before and after adding SMX; $\left[Q_{t}\right]$ is the concentration of quenching agent SMX, M; $\tau_{0}$ was the average lifetime of fluorescence of biomolecules in the absence of quenching agent, generally for $1 \times 10^{-8} \mathrm{~s}$. 
Table S1. The contents of proteins, polysaccharides and humic acids, and the elemental composition of the extracted EPSs from anammox granules.

\begin{tabular}{|c|c|c|c|c|c|c|c|}
\hline \multirow[t]{2}{*}{ proteins } & \multirow{2}{*}{$\begin{array}{c}\text { polysaccharides } \\
\mathrm{mg} \mathrm{g}^{-1} \mathrm{VSS}\end{array}$} & \multirow{2}{*}{$\begin{array}{c}\text { humic } \\
\text { acid }\end{array}$} & \multicolumn{5}{|c|}{ elemental composition (weight-\%) } \\
\hline & & & $\mathrm{C}$ & $\mathrm{O}$ & $\mathrm{N}$ & $\mathrm{H}$ & $\mathrm{S}$ \\
\hline $51.9 \pm 4.1$ & $9.1 \pm 1.5$ & $28.2 \pm 2.3$ & 31.8 & 27.1 & 9.7 & 7.2 & 3.6 \\
\hline
\end{tabular}

Table S2. The zeta potential of EPSs after interacting with different concentrations of SMX

$$
\text { Sample Zeta potential }(\mathrm{mV})
$$

EPS $100 \mathrm{mg} \mathrm{L}^{-1}$

$-14.22 \pm 1.01$

EPS 100mg L-1 + SMX $1 \mathrm{mg} \mathrm{L}^{-1}$

$-12.03 \pm 1.30$

EPS 100mg L-1 + SMX $2.5 \mathrm{mg} \mathrm{L}^{-1}$

$-6.88 \pm 1.08$

EPS 100mg L-1 + SMX 5 mg L-1

$-2.94 \pm 0.84$

EPS 100mg L-1 + SMX $10 \mathrm{mg} \mathrm{L}^{-1}$

$-0.37 \pm 0.27$

EPS 100mg L-1 + SMX $20 \mathrm{mg} \mathrm{L}^{-1}$

$-20.41 \pm 3.02$

Table S3. Binding constants of EPSs and SMX under different pH conditions

$\begin{array}{ccccccc}\mathrm{pH} & 5 & 6 & 7 & 8 & 9 & 10 \\ \mathrm{~K}\left(10^{4} \mathrm{M}^{-1}\right) & 0.01 & 0.30 & 15.26 & 1.99 & 1.99 & 0.93 \\ & & & & & & \\ \mathrm{R}^{2} & 0.9128 & 0.9905 & 0.9772 & 0.9914 & 0.9834 & 0.9933\end{array}$


Table S4. 2DCOS results on the sign of each cross-peak in synchronous and asynchronous maps of EPSs with SMX binding.

\begin{tabular}{cccccc}
\hline position & \multicolumn{5}{c}{ sign } \\
nm & 3409 & 1639 & 1402 & 1145 & 875 \\
3409 & + & $+(-)$ & $+(-)$ & $+(-)$ & $+(-)$ \\
1639 & + & $+(+)$ & $+(+)$ & $+(-)$ \\
1402 & & & & $+(-)$ & $+(-)$ \\
1145 & & & & + & $+(-)$ \\
875 & & & & + \\
\hline
\end{tabular}

Table S5. The free energy of a single amino acid residue that Amt domain interacted with SMX.

\begin{tabular}{ccccc}
\hline Residues & MM Energy & Polar Energy & Apolar Energy & Total Energy \\
ASN-90 & -4.6443 & 4.4158 & -0.2964 & -0.5172 \\
LEU-92 & -1.5967 & 0.701 & -0.0078 & -0.9022 \\
PHE-96 & -3.0007 & 1.2843 & -0.2436 & -1.9595 \\
TRP-144 & -4.2123 & 2.2085 & -0.2313 & -2.2376 \\
LEU-147 & -11.8364 & 8.4427 & -1.3707 & -4.7632 \\
PHE-148 & -4.9395 & 1.7468 & -0.1412 & -3.3335 \\
PRO-150 & -0.6667 & 0.0181 & -0.1016 & -0.7502 \\
ASP-163 & -3.1479 & 4.2815 & -0.0089 & 1.1208 \\
ALA-165 & -1.1819 & 0.6623 & -0.0127 & -0.5322 \\
GLY-226 & -0.8686 & 1.6168 & -0.038 & 0.712 \\
SER-227 & -7.4547 & 8.6864 & -0.5542 & 0.6815 \\
LEU-228 & -3.462 & 2.2495 & -0.2601 & -1.4684 \\
LEU-229 & -7.2648 & 0.7209 & -0.4939 & -7.0332 \\
ARG-230 & -18.3614 & 21.639 & -0.4632 & 2.7867 \\
\hline
\end{tabular}



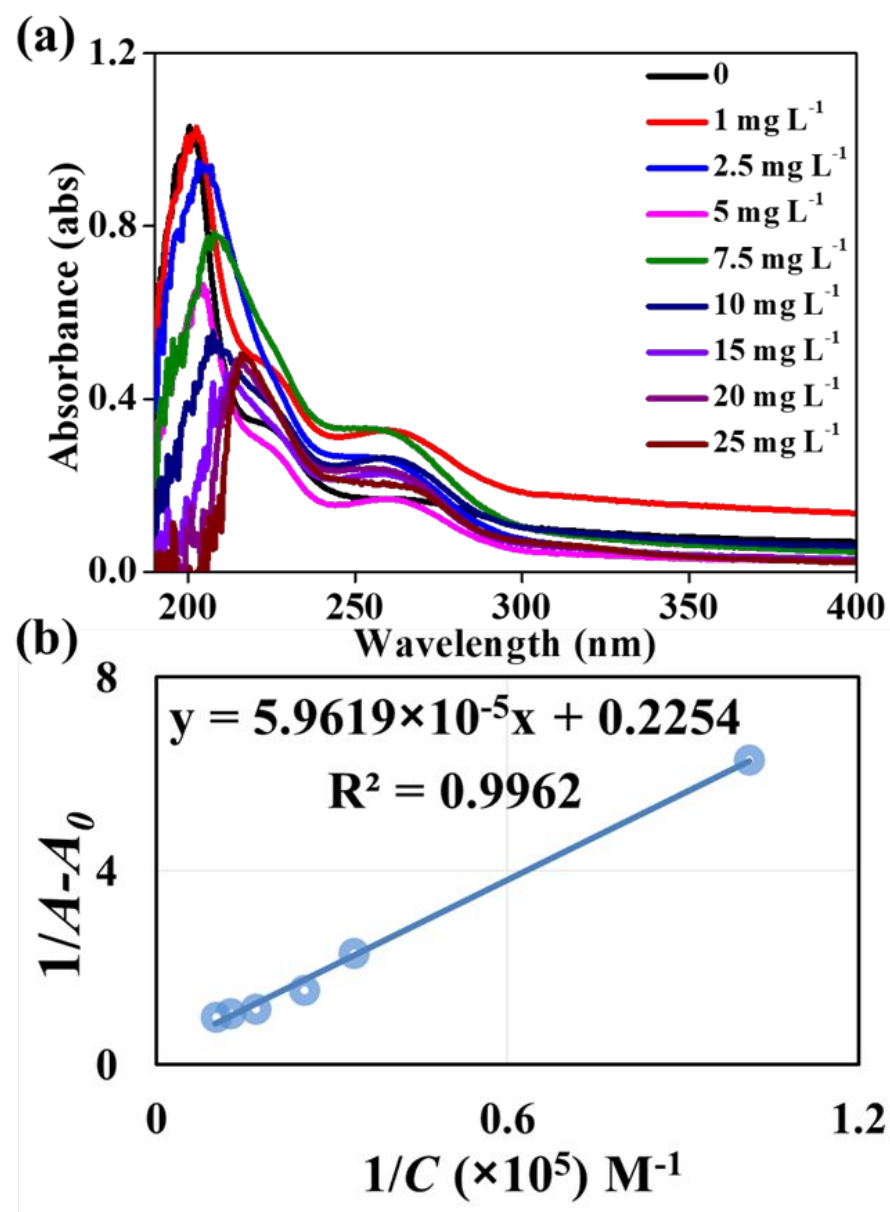

Figure S1. The UV differential absorption spectrum of EPSs-SMX mixture (a) and the linear regression of the binding constant obtained according to the double reciprocal formula (b). 

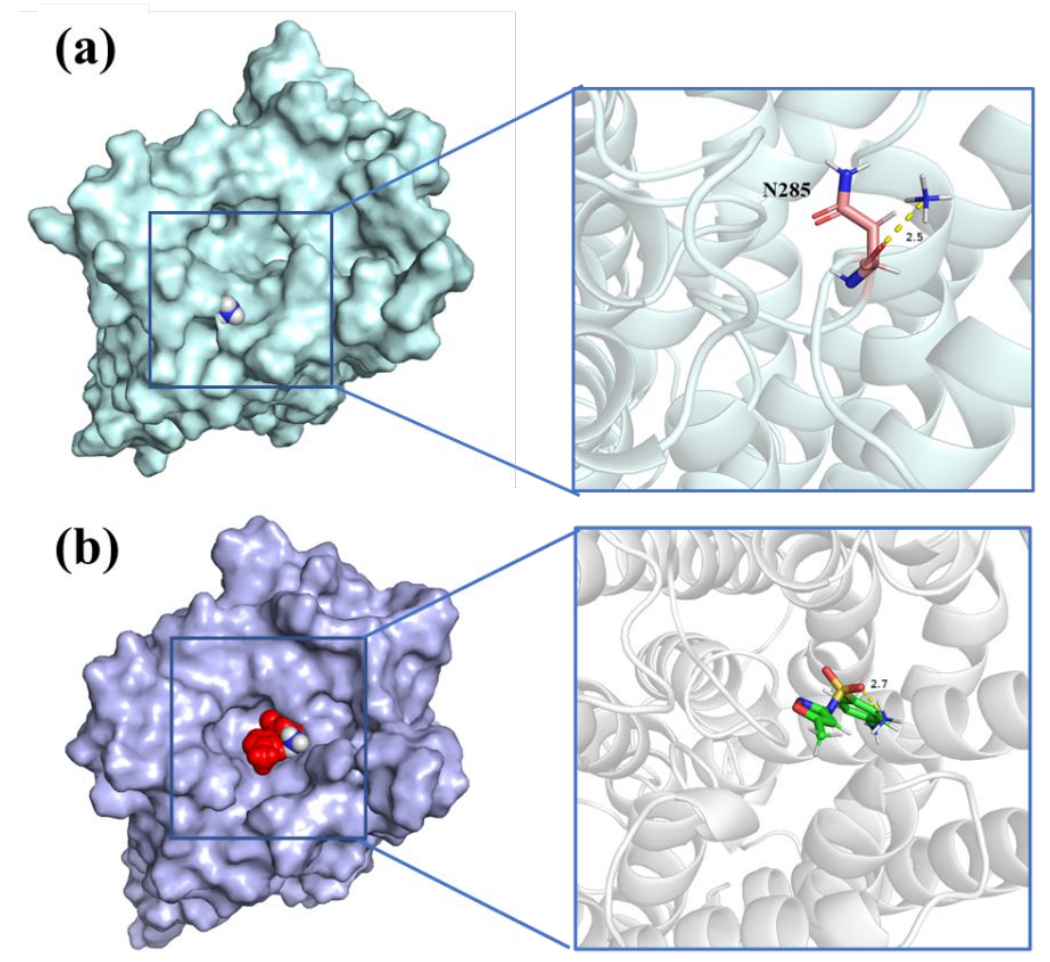

Figure S2. The docking conformation of Amt domain and $\mathrm{NH}_{4}^{+}$(a) and Amt domainSMX and $\mathrm{NH}_{4}^{+}(b)$. 


\section{SUPPORTING REFERENCE}

1. Chen, Z.; Meng, Y.; Sheng, B.; Zhou, Z.; Jin, C.; Meng, F., Linking exoproteome function and structure to anammox biofilm development. Environ. Sci. Technol. 2019, 53, (3), 1490-1500. 